Determinación de las características hidrodinámicas de dos sedimentadores sedhelcon para el tratamiento de aguas residuales.

Determining hydrodynamic characteristics of two sedhelcon settlers for sewage treatment.

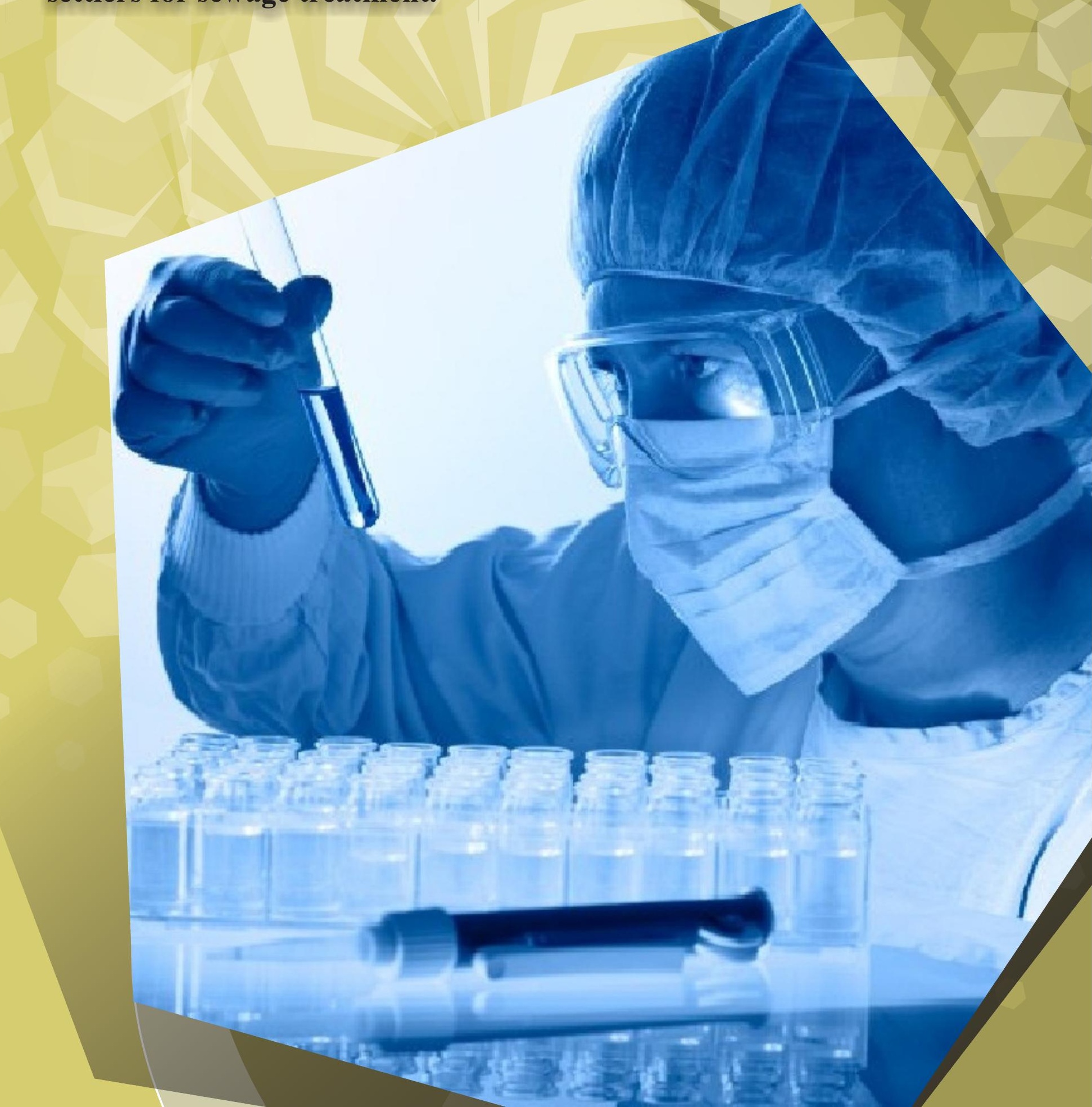




\title{
Determinación de las características hidrodinámicas de dos sedimentadores sedhelcon para el tratamiento de aguas residuales ${ }^{1}$. Determining hydrodynamic characteristics of two sedhelcon settlers for sewage treatment.
}

\author{
Ángel Camilo Pascal Suarez², Juan Pablo Rodríguez Miranda ${ }^{3}$. \\ ${ }^{2,3}$ Universidad Distrital Francisco José de Caldas.
}

Artículo recibido en febrero de 2016; artículo aceptado en abril de 2016

Citación del artículo: Pascal, A. Rodríguez. J. (año). Determinación De Las Características Hidrodinámicas De Dos Sedimentadores Sedhelcon Para El Tratamiento De Aguas Residuales. I+D Revista de Investigaciones, 7 (1), 117 -124.

\begin{abstract}
Resumen
Las características hidrodinámicas de un sedimentador o un reactor en general son determinantes para poder evaluar los diferentes aspectos de funcionamiento y del comportamiento del flujo de agua en los mismos, así; dentro de los sedimentadores se presenta reacciones que por lo general son de tipo cinético de transporte del agua, es decir un fenómeno netamente físico. Dentro del diseño de un sistema de tratamiento existen factores que determinan y limitan la construcción y el diseño de los mismo, por tal motivo es necesario hacer una reevaluación de las diferentes variables de los procesos en condiciones reales de operación. El presente artículo presenta los resultados de un modelo de sedimentador de flujo helicoidal mediante la aplicación de los diferentes modelos matemáticos como: modelo matemático wolf-resnick, indice de dispercion de morris (idm) y la curva de tendencia, estos métodos se analizaron aplicando el método de trazadores para los mismos.
\end{abstract}

Palabras clave: agua residual, sedimentador, remoción.

Abstract
The hydrodynamic characteristics of a settler or a
reactor generally are crucial to evaluate different aspects of
performance and the behavior of water flow therein, and;
within the sedimentation reactions are usually type of
kinetic transport of water, ie a purely physical phenomenon
occurs. Within the design of a treatment system there are

factors that determine and limit the construction and design of the same, for this reason it is necessary to re-evaluation of the different process variables in real operating conditions. This paper presents the results of a model settler helical flow through the application of different mathematical models as mathematical model WOLF-Resnick, INDEX dispersal MORRIS (IDM) and the trend curve, these methods were analyzed using the tracers method therefor.

Keywords: wastewater, sedimentation, removal.

\section{Introducción}

Para la remoción de partículas sedimentables, suspendidas y una fracción de la materia orgánica, la sedimentación en las aguas residuales es una operación considerada en dos fases (líquido y sólido), tanto en las fases primaria, intermedia y secundaria, como complemento óptimo para favorecer los procesos biológicos de tratamiento aeróbico (Simate, 2015; Romero, 2004; Nocón, 2016), dentro del flujo de la masa de agua se presentan propiedades propias de la partícula a sedimentar (forma, tamaño, densidad, permeabilidad, según el tipo del agua residual), el grado de aglomeración, la tasa de sedimentación entre otros, son factores que dentro del tanque sedimentación influyen en la eficiencia en lo que respecta a la remoción de las partículas contenidas en el mismo (Hribersek, 2011; Burger, 2015), estableciendo en alguna medida el grado de homogeneidad posible en las fases líquido - sólido y que convencionalmente, se podria

\footnotetext{
${ }^{1}$ Artículo investigación con enfoque cuantitativo, resultado de un proyecto de investigación terminado, perteneciente al área de Ingeniería Ambiental, tratamiento de aguas residuales, desarrollado en el grupo de investigación: AQUAFORMAT, Financiado por la Universidad Distrital Francisco José de Caldas, Bogotá D.C., Colombia.

${ }^{2}$ Tecnólogo en Saneamiento Ambiental. Universidad Distrital Francisco José de Caldas. Facultad del Medio Ambiente y Recursos Naturales. Semillero de Investigación OBATALÁ. Bogotá-Colombia Correo electrónico: camilo.pascal@gmail.com

${ }^{3}$ Rodríguez Miranda Juan Pablo Profesor Asociado. Facultad del Medio Ambiente y Recursos Naturales. Universidad Distrital Francisco José de Caldas. Grupo de investigación: AQUAFORMAT. Correo electrónico: jprodriguezm@udistrital.edu.co
} 
analizar es una sola dimensión, considerando la descripción de la concentración de sólidos en función de la profundidad del tanque y generando una tendencia hiperbólica no lineal (Ruiz, 2015; Kynch, 1952; Ivar, 2001).

De acuerdo a lo anterior, el conocer los aspectos hidrodinámicos que se desarrollan en los tanques de sedimentación y en especial en los tanques SEDHELCON (sedimentación helicoidal pirámide truncada cónico, para la remoción de partículas en suspensión o flóculos con alta velocidad de sedimentación, se recurre al uso de sedimentadores de flujo helicoidal) en relación con el tiempo de retención, tipo de flujo, espacios muertos, cortocircuitos entre otros (Giacoman, 2003), son factores determinantes con el comportamiento hidráulico y de efectividad en la remoción del tipo de partículas señaladas anteriormente generando en algunos casos biofloculación y bajo sustrato de carga, afectando procesos biológicos posteriores (Fan, 2007; Rodríguez, 2008; Amanatidou, 2015).

El presente trabajo, considera el análisis del comportamiento del flujo del agua en un tanque de sedimentador SEDHELCON, el cual puede utilizar métodos tales como el modelo matemático de Wolf Resnick, índice de dispersión de Morris (IDM) y el análisis de la curva de tendencia; la ventaja que presentan estos métodos, es que permiten obtener una caracterización del comportamiento del agua en cada uno de los componentes que configuran el sistema de tratamiento, así como poder identificar el porcentaje de zonas muertas, zonas de corto circuito, tipos de flujo entre otros; Adicional además presenta la correlación de la velocidad de sedimentación con la temperatura del agua presente en el tanque sedimentador.

\section{Tipo de Investigación.}

El tipo de investigación aplicada a este trabajo -es del tipo experimental,- debido a que permite (en condiciones de control riguroso) establecer con seguridad las relaciones causa - efecto y tener en consideración los factores que pueden afectar el experimento (Vergel G., 2010); además según el tiempo de ocurrencia de los hechos y el registro de la información relacionada con el tema de estudio, el tipo de investigación aplicada se considera como retrospectiva, con el propósito de alcanzar conocimientos (Vergel G., 2010).

\section{Instrumentos y procedimientos:}

Los reactores de sedimentación, están constituidos por un sedimentador primario Sedhelcon4 y un sedimentador secundario también denominado Sedhelcon, cada uno operando con caudales de $0.2 \mathrm{~L} / \mathrm{min}$ (Rodríguez J., 2013).

Se realizó un estudio descriptivo - prospectivo a partir de la información proveniente de los datos de operación de los sedimentadores y se realizó un analisis estocástico, en términos de los datos resultantes. Para realizar este trabajo, la metodología aplicada se dividió en fases:

Fase I. Prueba de trazadores aplicada a los dos sedimentadores Sedhelcon.

Fase II. Análisis e interpretación de los datos obtenidos en la fase I, por los modelos matemáticos Wolf Resnick, IDM y Curva De Tendencia.

Fase III. Análisis, interpretación y gráficas resultantes de los resultados de los modelos matemáticos Wolf Resnick, IDM Y Curva De Tendencia.

Además, se realizó un análisis descriptivo para la determinación de la ecuación de velocidad de sedimentación (ambos sedimentadores, primario $\mathrm{y}$ secundario) mediante el método de regresión lineal de mínimos cuadrados y fue comparado con los criterios establecidos por la literatura especializada.

Tabla 1.

Criterios de diseño de sedimentadores.

\begin{tabular}{|c|c|c|}
\hline $\begin{array}{c}\text { TANQUE DE } \\
\text { SEDIMENTACION }\end{array}$ & $\begin{array}{c}\text { TASA DE } \\
\text { SEDIMENTACION } \\
\left(\mathrm{m}^{3} / \mathrm{m}^{2} \text { día }\right)\end{array}$ & AUTOR \\
\hline Primario & $\begin{array}{l}32-48 \text { (Caudal Medio) } \\
80-120 \text { (Caudal Pico) }\end{array}$ & RAS 2000 \\
\hline Primario & $\begin{array}{c}32-49 \text { (Caudal Medio) } \\
80-122 \text { (Caudal Pico) }\end{array}$ & WEF. 1998 \\
\hline Primario & $\begin{array}{l}33-49 \text { (Caudal Medio) } \\
81-122 \text { (Caudal Pico) }\end{array}$ & $\begin{array}{c}\text { MELCALF. } \\
1991\end{array}$ \\
\hline Primario & $\begin{array}{l}33-49 \text { (Caudal Medio) } \\
81-122 \text { (Caudal Pico) }\end{array}$ & $\begin{array}{c}\text { MELCALF. } \\
1991\end{array}$ \\
\hline Primario & $30-45$ (Caudal Medio) & $\begin{array}{c}\text { CRITETENDE } \\
\text { N, } 2005\end{array}$ \\
\hline Secundario & $\begin{array}{c}16-32 \text { (Caudal Medio) } \\
40-48 \text { (Caudal Pico) }\end{array}$ & RAS 2000 \\
\hline Secundario & $\begin{array}{c}16-29 \text { (Caudal Medio) } \\
40-65 \text { (Caudal Pico) }\end{array}$ & WEF. 1998 \\
\hline Secundario & $\begin{array}{l}8-33 \text { (Caudal Medio) } \\
24-49 \text { (Caudal Pico) }\end{array}$ & $\begin{array}{l}\text { MELCALF. } \\
1991\end{array}$ \\
\hline
\end{tabular}

Fuente: Los autores

Análisis de resultados y discusiones.

El análisis del comportamiento del flujo del agua en un tanque de sedimentador SEDHELCON para el tratamiento del agua residual doméstica, se estableció de la siguiente forma:

PruebaDe Trazadores.

\footnotetext{
${ }^{4}$ Es un sedimentador con forma de pirámide truncada, con canales laterales con el fin de efectuar un flujo helicoidal y facilitar la sedimentación de las partículas al interior de este y poder conseguir el tratar aguas con alto contenido de materiales en suspensión o flóculos con alta velocidad de sedimentación en flujo helicoidal.
} 
Las limitantes de los diseños de sistemas de tratamiento presentan el supuesto, de que el flujo en los reactores y/o sedimentadores es de dos tipos: en pistón y en mezcla completa. En realidad el flujo en los reactores no se ajusta exactamente a estas situaciones ideales (Giacoman, 2003). En muchas ocasiones, el comportamiento de los flujos es parecido al de los sistemas idealizados, por tanto se puede suponer que el reactor se comporta como la suma de estos reactores, sin que se esté incurriendo en errores grandes. Igualmente, se puede presentar el caso en el que se den canalizaciones del flujo por recirculación del fluido, o por formación de zonas muertas, lo cual genera reducción de la eficiencia del sistema.

Para comprender el funcionamiento de un sedimentador desde el punto de vista hidráulico, es conveniente utilizar una sustancia trazadora (sal, colorantes, ácidos o una sustancia radioactiva) que pueda ser aplicada en la entrada del sedimentador y que simultáneamente se empiece a registrar la concentración del trazadora la salida del sedimentador por intervalos de tiempo determinados. (Vargas, 2006).

Para poder visualizar el funcionamiento de una unidad desde el punto de vista hidráulico, es conveniente utilizar una sustancia trazadora (sal, colorantes, ácidos o una sustancia radioactiva), que puede ser aplicada en la entrada del sedimentador $\mathrm{y}$, simultáneamente se registran los concentraciones del trazador a la salida del mismo. (Vargas, 2006) La cantidad de trazador a utilizar "P" que se necesita para añadir a los dos sedimentadores está dado por la siguiente ecuación: (Vargas, 2006)

$$
P=\frac{\forall * K * C_{O}}{I * 10^{3}}
$$

Donde:

$\mathrm{P}=$ masa del trazador por añadir al sedimentador.

$\forall=$ volumen útil del sedimentador $\left(\mathrm{m}^{3}\right)$.

$\mathrm{K}=$ constante de corrección.

$\mathrm{Co}=$ concentración del trazador $\left(\mathrm{mg} / \mathrm{Log} / \mathrm{m}^{3}\right)$

$\mathrm{I}=$ grado de pureza del trazador.

Como sustancia trazadora se decidió utilizar el cloruro de sodio $(\mathrm{NaCl})$, debido a su fácil adquisición así como su detección por medio de la conductividad, en el agua. Así mismo, sabiendo que los sólidos disueltos totales tiene una relación directa con la conductividad, por estar relacionados con las sales que pueda poseer el agua, se decidió utilizar una concentración que fuera apreciable de determinar y en la cual los sólidos disueltos del agua residual no interfieran, por lo que utilizó una concentración igual a $2000 \mathrm{mg} / \mathrm{L}$. La constante $\mathrm{K}$ se determinó en virtud de la masa molecular del trazador:

$$
\text { Por lo tanto: } \quad K=\frac{\text { masa de } \mathrm{NaCI}}{\text { masa de } C I}
$$

$$
K=\frac{23 \mathrm{~g} / \mathrm{mol}+35.5 \mathrm{~g} / \mathrm{mol}}{35.5 \mathrm{~g} / \mathrm{mol}}
$$

Esta constante se implementa para determinar la cantidad de trazadora "P" a utilizar para el sedimentador primario:

$$
K=\frac{0.26 * 1.65 * 2000}{0.99 * 10^{3}}=0.89 \mathrm{Kg}=866 \mathrm{~g} .
$$

El volumen de agua a utilizar para poder diluir la cantidad de trazador se determinó así:

$$
V_{1} * C_{1}=V_{2} * C_{2}
$$

Donde:

$\mathrm{V} 1=$ volumen del sedimentador

$\mathrm{C} 1=$ concentración (valor promedio) del parámetro TDS.

$\mathrm{V} 2=$ Valor a determinar

$\mathrm{C} 2=$ concentración de trazador.

Despejando V2 de la ecuación y reemplazando:

$$
K=\frac{0.26 \mathrm{~m}^{3} * \frac{1000 \mathrm{~L}}{\mathrm{~m}^{i}} * 173 \mathrm{mg} / \mathrm{L}}{2000 \mathrm{mg} / \mathrm{L}}=11.50 \mathrm{~L}
$$

Para un sedimentador primario con un volumen de 0.26 $\mathrm{m}^{3}$ se debe diluir 866 gr de trazador con $22.50 \mathrm{~L}$. de agua.Tal como se procedió con el sedimentador primario se procede con el secundario: Cantidad de trazador "P":

$$
P=\frac{0.40 * 1.65 * 2000}{0.99 * 10^{3}}=1.33 \mathrm{Kg}=1333 \mathrm{~g} .
$$

$$
V_{2}=\frac{0.40 m^{3} * \frac{1000 L}{m^{3}} * \frac{1880 m g}{L}}{2000 \frac{m g}{g}}=36 L
$$

Por lo tanto para el sedimentador secundario de volumen $0.40 \mathrm{~m}^{3}$ se debe diluir $1333 \mathrm{gr}$ del trazador con $36 \mathrm{~L}$. de agua. Con los datos anteriormente obtenidos, se procedió a aplicar el trazador en cada uno se los sedimentadores en un punto en el que este se pudiese mezclar de forma instantánea, y proceder a la toma de datos de conductividad, generando una curva de tendencia del comportamiento del agua en cada uno de los sedimentadores.

\section{Curva de tendencia sedimentador primario}

Con los datos obtenidos del método de trazadores se procedió a determinar la conductividad en el efluente de cada uno de los sedimentadores, graficando los datos obtenidos de las concentraciones versus el tiempo entre cada toma de datos de la conductividad. De lo anterior se 
obtiene las siguientes figuras de curva de tendencia:

Figura1. Curva de tendencia sedimentador primario

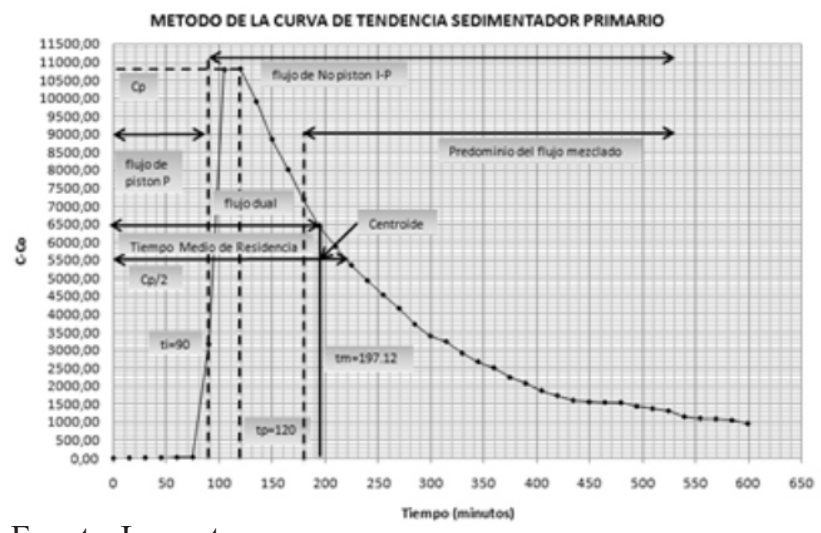

Fuente: Los autores

De la figura de la curva del sedimentador primario se puede decir:

$\mathrm{ti}=90$ minutos

$\mathrm{tp}=120$ minutos

$\mathrm{tm}=197,12$ minutos

to $=1300$ minutos

$\mathrm{tf}=\quad 600$ minutos

Donde:

ti: tiempo en que aparece el trazador en el efluente.

tp: presentación de la máxima concentración.

tm: tiempo del paso del 50\% de trazador.

to: tiempo teórico de retención.

tf: tiempo en que pasa la totalidad del trazador.

Analizando los criterios anteriormente obtenidos, según (Vargas, 2006)se obtiene:

Tabla 2.

Tipo de flujo del sedimentador primario.

\begin{tabular}{cc}
\hline CRITERIO & CONSECUENCIA \\
\hline$t i /$ to $\therefore 90 / 1300=0,07$ & $\begin{array}{c}\text { Predominio flujo } \\
\text { mezclado }\end{array}$ \\
$t m /$ to $\therefore 197,12 / 1300=0,15$ & $\begin{array}{c}\text { Existencia de } \\
\text { cortocircuitos } \\
\text { hidráulicos }\end{array}$ \\
$\mathrm{e}=\frac{(600-120)-(120-90)}{1300}=0.3$ & $\begin{array}{c}\text { Existencia del flujo } \\
\text { mezclado }\end{array}$ \\
\end{tabular}

Fuente: Los autores

Curva de tendencia sedimentador secundario.
Con los datos obtenidos de trazadores en el sedimentador secundario se procedió a realizar y analizar la curva de tendencia en la siguiente gráfica:

Figura 2. Curva de tendencia sedimentador secundario

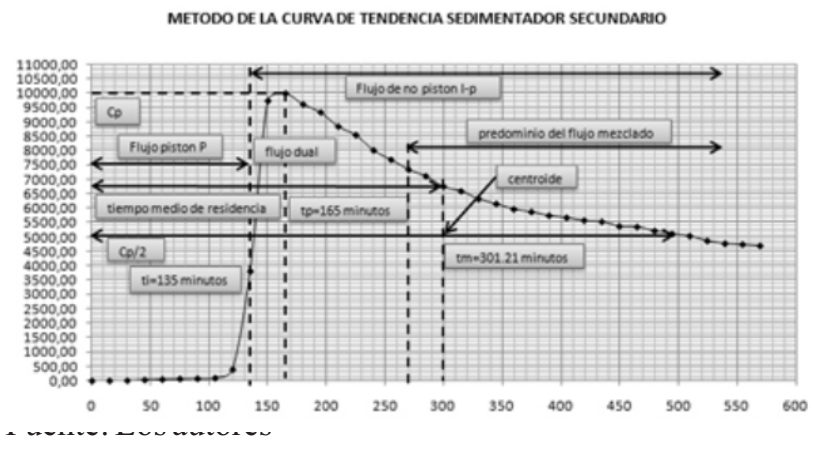

De la figura 2 de la curva del sedimentador secundario se puede deducir:

$\mathrm{ti}=135$ minutos

$\mathrm{tp}=165$ minutos

$\mathrm{tm}=301.21$ minutos

to $=2000$ minutos

$\mathrm{tf}=570$ minutos

Analizando los criterios anteriormente obtenidos, según Canepa de Vargas se obtiene:

Tabla 3.

Tipo de flujo del sedimentador primario

\begin{tabular}{|c|c|}
\hline CRITERIO & CONSECUENCIA \\
\hline ti $/$ to $\therefore 135 / 2000=0,067$ & $\begin{array}{l}\text { Predominio flujo } \\
\text { mezclado }\end{array}$ \\
\hline $\mathrm{tm} / \mathrm{to} 301,21 / 2000=0,15$ & $\begin{array}{l}\text { Existencia de } \\
\text { cortocircuitos } \\
\text { hidráulicos }\end{array}$ \\
\hline$t p / t o \therefore 165 / 2000=0,0825$ & $\begin{array}{l}\text { Predominio del } \\
\text { flujo mezclado }\end{array}$ \\
\hline$\frac{(570-165)=(165-135)}{2000}=0.19$ & $\begin{array}{l}\text { Existencia del } \\
\text { flujo mezclado }\end{array}$ \\
\hline
\end{tabular}

Índice de dispersión de Morris (IDM) sedimentador primario.

Físicamente, esta curva representa un aumento de la concentración de trazador en la corriente de salida del reactor. (Molina, 2010)Al acumular los datos sobre la cantidad de trazador que pasa, expresado en porcentajes y dibujados en papel que tenga escala de probabilidades en las abscisas y escala logarítmica en las ordenadas para 
diferentes tiempos, se obtiene aproximadamente una línea recta. (Vargas, 2006)El segmento comprendido entre el $10 \%$ y el $90 \%$ es el más regular y por eso Morris sugirió que la relación entre uno y otro se tomara como índice de dispersión. (Vargas, 2006). Así:

$$
\text { Indice de Morris }=\frac{\text { tiempo en que pasa el } 90 \%}{\text { tiempo en que pasa el } 10 \%}
$$

Si todo el flujo fuera de pistón, la curva que se presenta sería una línea horizontal, y el índice de Morrill sería 1, pues todo el trazador saldría en un tiempo $(\mathrm{t}=\mathrm{to})$ y nada saldría antes. (Carrión, 1992).

Figura 3. Indice de Morrill sedimentador primario

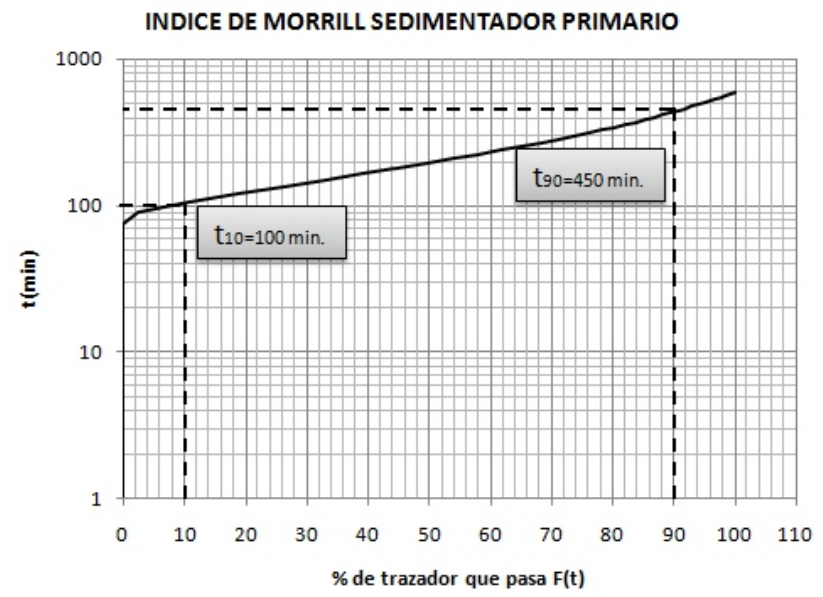

ruente: Los autores

Tabla 4

Índice de desempeño sedimentador primario.

$I_{M}=\frac{t_{90}}{t_{10}}=\frac{450}{100}=4,5$

\begin{tabular}{ccc}
\hline INDICE & TERMINO & VALOR. SED. 1 \\
\hline $\begin{array}{c}\text { Indice de } \\
\text { dispersion de } \\
\text { morrill (MDI). } \\
\text { Eficiencia } \\
\text { volumetrica } \\
\text { indice de }\end{array}$ & Im $=$ t90/t10 & $\mathrm{Im}=450 / 100=4,5$ \\
$\begin{array}{c}\text { corto circuito } \\
\text { indice de tiempo } \\
\text { de retencion } \\
\text { modal }\end{array}$ & tp/to 100 & $(1 / 4,5)^{*} 100=22,2 \%$ \\
\hline
\end{tabular}

Fuente: Los autores

Tabla 5
Índice de desempeño sedimentador secundario.

$I_{M}=\frac{t_{90}}{t_{10}}=\frac{500}{180}=2,8$

Figura 4. Indice de Morrill sedimentador secundario

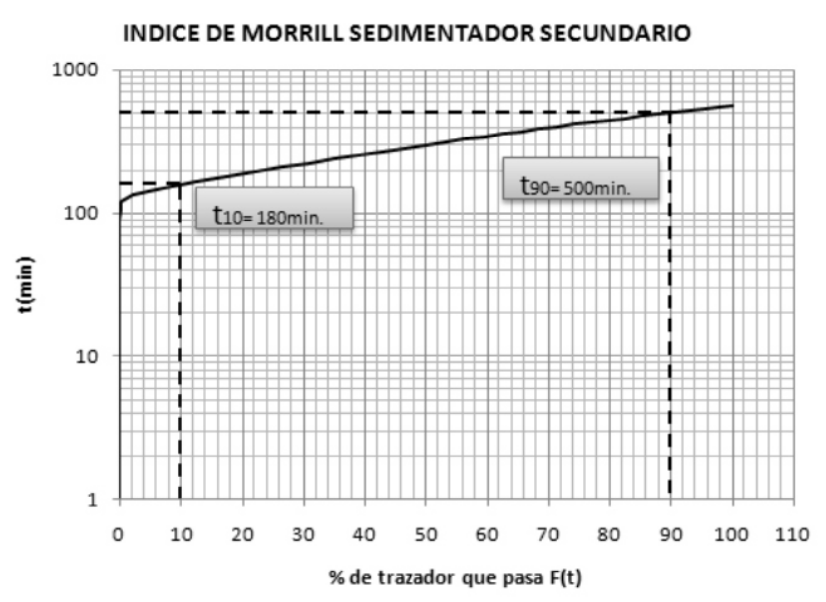

A medida que hay más proporción de flujo mezclado, aumenta el ángulo que la curva hace con la horizontal, pues hay una distribución más amplia del tiempo de retención. (Carrión, 1992)El estudio de la totalidad de la curva puede suministrar información más completa de la curva de tendencia. (Carrión, 1992)

\section{Modelo matemático Wolf y Resnick. (Sedimentador primario)}

Una de las formas para determinas las características hidráulicas de un sedimentador es el método Wolf Resnick, este consiste en ajustar una línea tangente por la parte recta de la curva, la pendiente de esta recta es la tan $\alpha$ y su intersección con el valor de 1.0 en las ordenadas permite calcular el valor de $\boldsymbol{\theta}$. (Vargas, 2006)En las Gráficas 5 y 6 se observan lasrectas tangentes de los dos sedimentadores.

Figura 5. Curva tangente del sedimentador primario

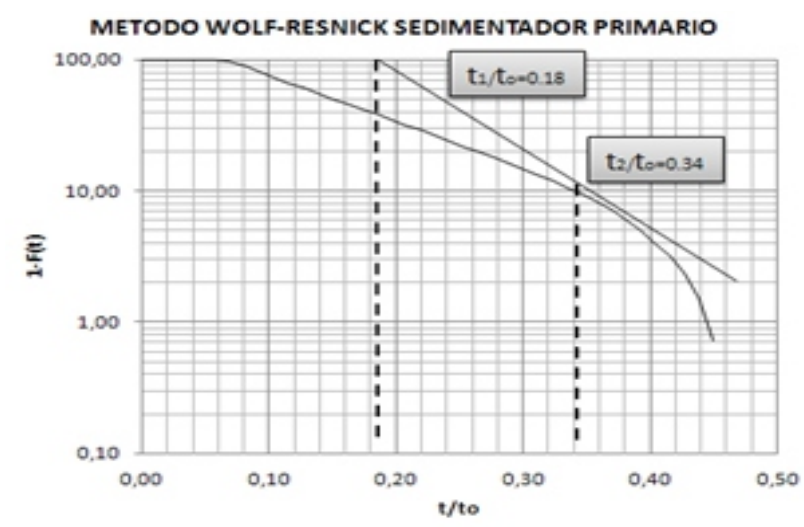

I+D Revista de Investigaciones ISSN 22561676 Volumen 7 Número 1 Año 04 Enero - Junio de 2016 p.p 117-124. 


\begin{tabular}{|c|c|c|}
\hline INDICE & TERMINO & VALOR. SED. 1 \\
\hline $\begin{array}{c}\text { Indice de } \\
\text { dispersion de } \\
\text { morrill (MDI). }\end{array}$ & $\operatorname{Im}=\mathrm{t} 90 / \mathrm{t} 10$ & $\operatorname{Im}=500 / 180=2,77$ \\
\hline $\begin{array}{l}\text { Eficiencia } \\
\text { volumetrica }\end{array}$ & $(1 / \mathrm{IDM}) * 100$ & $(1 / 2,77) * 100=36,10 \%$ \\
\hline $\begin{array}{l}\text { indice de } \\
\text { corto circuito }\end{array}$ & ti/to & $135 / 2000=0,0675$ \\
\hline $\begin{array}{l}\text { indice de tiempo } \\
\text { de retencion } \\
\text { modal }\end{array}$ & tp/to & $165 / 2000=0,0825$ \\
\hline
\end{tabular}

Figura 6. Curva tangente del sedimentador secundario.

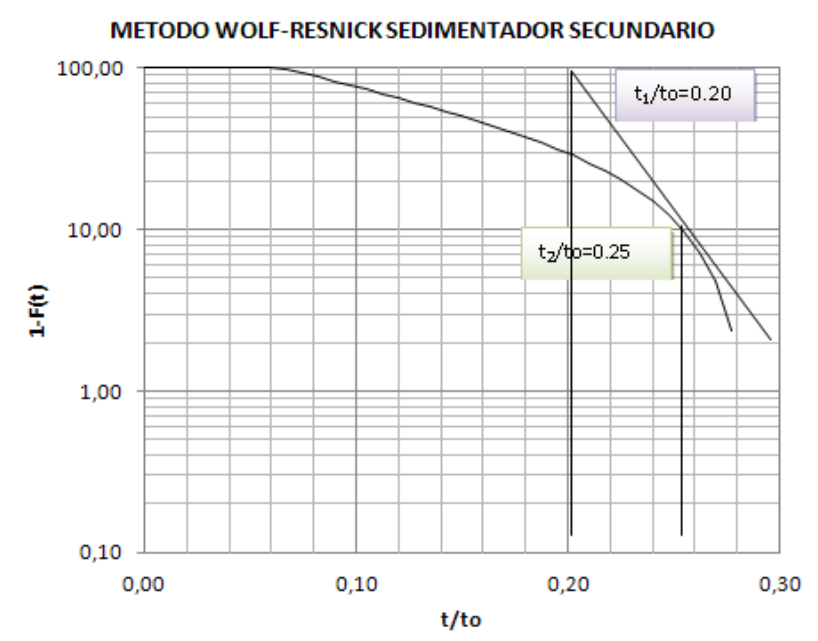

ruente: Los autores

Tangente de inclinación y tipo de flujos, según Canepa de Vargas (2005):

Remplazando valores $\frac{1}{\frac{t_{2}}{t_{n}}-\frac{t_{1}}{\text { la etuación anterior tenemos: }}}$

$$
\frac{1}{0.34-0.18}=\frac{1}{0.16}=6.25
$$

Determinación del flujo predominante, según Canepa de Vargas (2005):

\section{A. Flujo pistón:}

$$
P=\frac{\left(\mathrm{t}_{1} / \mathrm{t}_{0}\right) *\left(\operatorname{tg}_{\propto} \text { inclinación }\right)}{\text { Loge }+\left(\frac{\mathrm{t}_{1}}{\mathrm{t}_{2}}\right) * \text { tg adnclinación }}
$$

Remplazando valores en la eccuación anterior tenemos:

$$
P=\frac{0.18 * 6.25}{0.435+0.18 * 6.25}=\frac{1.125}{1.56}=0.72=72 \%
$$

B. Flujo mezclado:

$$
(1-p)
$$

Remplazando valores en la ecuación anterior tenemos:

$$
(1-0.72)=0.28=28 \%
$$

Porcentaje de zonas muertas, según Canepa de Vargas (2005):

$$
P=1=\frac{\frac{t_{1}}{t_{0}}}{}
$$

Remplazando valores en fluichacedominante tenemos:

$$
m=1-\frac{0.18}{0.72}=0.75=75 \%
$$

Desarrollando estos mismo cálculos por el método Wolf 1 , 2, 3, 4 del sedimentador primario, estas se aplicaron para el sedimentador secundario para el Modelo matemático Wolf y Resnick (Chatzakis, 2006).

$$
I_{M}=\frac{t_{90}}{t_{10}}=\frac{500}{180}=2,8
$$

Tangente de inclinación:

$$
\frac{1}{\frac{t_{2}}{t_{0}}-\frac{t_{1}}{t_{0}}}=\frac{1}{0.25-0.20}=\frac{1}{0.05}=0.95
$$

Determinación del flujo predominante

A. Flujo pistón:

$$
\begin{gathered}
P=\frac{\left(\frac{\mathrm{t}_{1}}{\mathrm{t}_{0}}\right) * \text { tg } \propto \text { inclinación }}{\operatorname{Loge}+\left(\frac{\mathrm{t}_{1}}{\mathrm{t}_{0}}\right) * \text { tgøinclinación }} \\
P=\frac{0.20 * 0.95}{0.435+0.20 * 0.95}=\frac{0.19}{0.625}=0.304=30.4 \%
\end{gathered}
$$

B. Flujo mezclado:

$$
(1-p)=(1-0.304)=0.696=69.6 \%
$$

Porcentaje de zonas muertas:

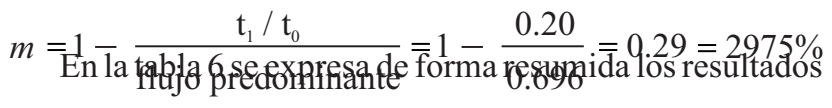


del modelo matemático Wolf Resnick.

Tabla 6

Tipos de flujo y zonas muertas de los sedimentadores.

\begin{tabular}{cccc}
\hline UNIDAD & FLUJO & FLUJO & ZONAS \\
& PISTÓN & MEZCLADO & MUERTAS \\
\hline $\begin{array}{l}\text { SEDIMENTADOR } \\
\text { PRIMARIO }\end{array}$ & $72 \%$ & $28 \%$ & $75 \%$ \\
$\begin{array}{l}\text { SEDIMENTADOR } \\
\text { FEERFENDAARLPes }\end{array}$ & $30,40 \%$ & $69,60 \%$ & $29 \%$ \\
\hline
\end{tabular}

Ecuaciones de velocidad de sedimentación de las partículas.

Se determinó la ecuación de velocidad de sedimentación de las partículas para los sedimentadores, primario y secundario, bajo el método de mínimos cuadrados debido a la tendencia de los datos; así como sus coeficientes de correlación o de Pearson. El coeficiente de correlación o de Pearson (r), determina el grado de relación existente entre dos variables. Cuando el coeficiente de correlación es igual a 1.0 se establece que hay una correlación perfecta, dado que los valores observados son exactamente iguales a los estimados (figuras 3 y 4 ); en otras palabras, los puntos en una figura (nube de puntos) se confunden con los de la recta. (Martínez, 2000).

Figura 7. Influencia de la temperatura sobre la velocidad de sedimentación, sedimentador primario.

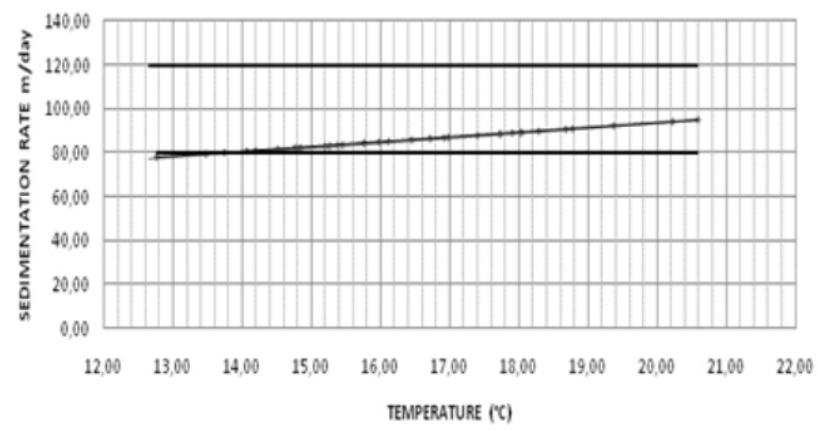

Figura8. Influencia de la temperatura sobre la velocidad de sedimentación, sedimentador secundario.

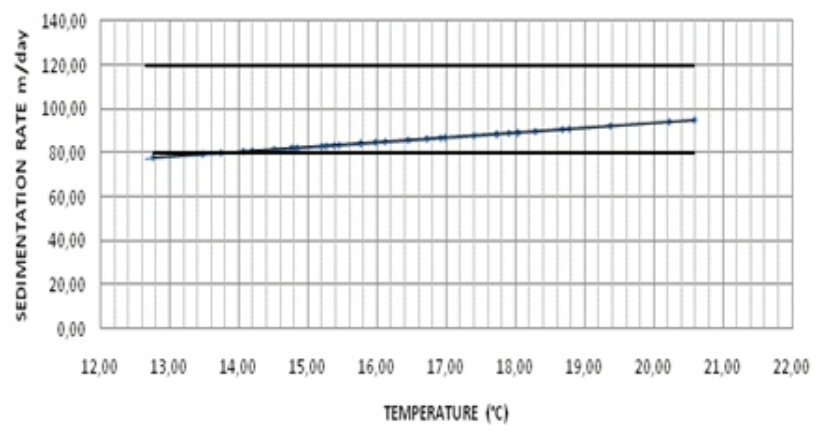

Para las figuras 7 y 8 , se determinó el coeficiente de correlación, dando como resultado para el sedimentador primario un coeficiente de correlación igual a $\mathrm{r}=0.99971 \mathrm{y}$ para el sedimentador secundario un coeficiente de correlación igual a $\mathrm{r}=0.99965$, los coeficientes de correlación de los dos sedimentadores son cercanos a 1.0 lo que se puede expresar como un tipo de correlación excelente;así mismo las ecuaciones de velocidad de sedimentación son:

- Sedimentador primario: $\mathrm{VsI}=2.1959 . \mathrm{T}+44.449$

- Sedimentrador secundario: VsII=2.2068.T+49.272

Dónde: VsIy VsII $=\left(\mathrm{m}^{3} / \mathrm{m}^{2}\right.$.dia $), \mathrm{T}=\left({ }^{\circ} \mathrm{C}\right)$.

La temperatura afecta el fenómeno de sedimentación, específicamente en la velocidad de sedimentación de las partículas, la tasa de sedimentación de sólidos en aguas con una mayor gradiente de temperatura-, es mayor que en aguas con un bajo gradiente de temperatura, esto debido al cambio de viscosidad del agua (Romero, 2004). Dentro de los criterios de diseño se establece una carga hidráulica superficial para los sedimentadores, esta carga para un sedimentador primario establece entre 80 y $120 \mathrm{~m}^{3} / \mathrm{m}^{2}$.dia; entonces para un sedimentador secundario se establece entre 40 y $65 \mathrm{~m}^{3} / \mathrm{m}^{2}$.dia. (Rodríguez J. , 2013)

\section{Conclusiones}

Las pruebas de trazadores y los modelos matemáticos son de gran importancia para poder determinar e interpretar el comportamiento de un sistema de tratamiento, y los elementos que intervienen para su correcto funcionamiento. Los modelos matemáticos aplicados para los dos sedimentadores ponen en manifiesto el predominio del flujo mezcla completa para el sedimentador primario, pero en el caso del sedimentador secundario, por el modelo Wolf Resnill presenta un flujo predominante de pistos, por el contrario por los modelos IDM y Curva de tendencia, este presenta un flujo predominante de mezcla completa.

Los sedimentadores, presentan zonas de cortos circuitos en la que parte del flujo de agua que entra a los sedimentadores los atraviesa rápidamente sin permanecer almacenado en ellos.

Asimismo se observa un alto porcentaje de zonas muertas, es decir aquellos puntos donde no se genera una mezcla de las partículas con toda la masa de agua, algunas de las causas de estos se debe al diseño y/o configuración del mismo. Los porcentajes de zonas muertas concernientes a cada sedimentador son demasiado elevadas, aún así esto no implica que el proceso de remoción y eficiencia de los sedimentadores no puedan ser buenos, por el contrario el no presentarse un buen comportamiento hidráulico no implica necesariamente malas remociones, dado que en los sedimentadores se obtuvieron excelentes porcentajes de remoción. Los tiempos hidráulicos de retención en los sedimentadores, muestran una significativa ventaja, pues a mayor tiempo de estancia del agua en el sedimentador, dará lugar a que las partículas sedimenten de forma más 
eficiente.

La velocidad de sedimentación en el sedimentador primario en cuanto a su carga hidráulica superficial serefiere, se encuentra de los criterios establecidos, a diferencia del sedimentador secundario el cual está por encima de los establecido; esto manifestándose en una velocidad de sedimentación mayor debido a que el paso de la partícula entre el reactor biológico y el sedimentador secundario no presento turbulencia y la partícula presento mejor forma y tamaño.Los coeficientes de correlación obtenidos para los dos sedimentador demuestran una relación directa existente entre la velocidad de sedimentación de las partículas y su temperatura, expresándose así en una correlación excelente(Rodríguez J. ,2013).

\section{Referencias}

Amanatidou, E. (2015). Evaluating sedimentation problems in activated sludge treatment plants operating at complete sludge retention time. water research, 20-29.

Burger, R. (2015). Discontinuous finite volume element discretization for coupled flowtransport problems arising in models of sedimentation. Journal of Computational Physics, 446-471.

Carrión, J. (1992).Mejoramiento de calidad del aguapara consumo humano.Lima: OPS/CEPIS.

Chatzakis, M. (2006). Sedimentation tanks through the age. IWA $1 \mathrm{St}$ International symposium on water and wastewater technologies in ancient civilizatinos (págs. 75 78). Iraklio, Greece: IWA.

Fan, L. (2007). Numerical simulation of secondary sedimentation tank for urban wastewater. Journal of the Chinese Institute of Chemical Engineer, 425-433.

Giacoman, G. (2003). Análisis de la hidrodinámica de un reactor flujo pistón. Ingeniería, 7-19.

Hribersek, M. (2011). Experimental and numerical investigations of sedimentation of porous wastewater sludge flocs. water research, 1729-1735.

Ivar, O. (2001). Biological lamella sedimentation used for wastewater treatment. Aquacultural Engineering, 115127.

Kynch, G. (1952). A theory of sedimentation. Birmingham: The University Birmingham.

Molina, J. (2010). Modelamiento de las condiciones hidraulicas de una planta compacta para el tratamiento de aguas residuales domesticas.MedellIn.

Nocón, W. (2016). Quantitative monitoring of batch sedimentation based on fractional density changes. Powder Technology, 1-6.

O., L. (1998). Ingenieria de las reacciones quimicas. barcelona: reverte.

Rodríguez, J. (2013). Aumento en la eficiencia de remoción de contaminantes mediante un reactor aeróbico modificado a flujo pistón y sedimentador de flujo helicoidal. Teoría y praxis investigativa, 54- 71.

Rodríguez, P. (2008). Flow models for rectangular sedimentation tanks. Chemical Engineering and Processing, 1705-1716.

Romero, J. (2004). Tratamiento de aguas residuales, teoria y principios de diseño.Bogotá: Escuela Colombiana de Ingeniería.

Ruiz, R. (2015). Numerical solution of a multidimensional sedimentation problem using finite volume-element methods. Applied Numerical Mathematics, 280-291.

sanchez. (2013).

Simate, G. (2015). The treatment of brewery wastewater for reuse by integration of coagulation/flocculation and sedimentation with carbon nanotubes sandwiched in a granular filter bed. Journal of Industrial and Engineering Chemistry, $1277-1285$.

Vargas, L. (2006). Análisis de flujo y factores que determinan los periodos de retención. LIMA: OPS/CEPIS. 


\section{Directrices para la presentación de artículos en I+D Revista de Investigaciones de la Universitaria de Investigación y Desarrollo -UDI-.}

La revista acepta reportes de investigaciones empíricas, teóricas, ensayos, trabajos de meta-análisis, revisiones críticas de literatura.

\section{Proceso de Envío, Arbitraje y Aceptación del Artículo}

Los artículos que se sometan a revisión para su publicación deben dirigirse y enviarse a $I+D$ Revista de Investigaciones, en medio digital, versión MS Word, al correo electrónico

revistadeinvestigaciones@udi.edu.co.Los artículos enviados deben ser originales y no hallarse en proceso de evaluación en otra publicación científica simultáneamente a su presentación en $I+D$ Revista de Investigaciones, razón por la cual es indispensable que los autores firmen una carta, suministrada por la revista, donde certifiquen lo anterior, la c ual podrá ser descargad a del li n k http://www.udi.edu.co/investigaciones/102-revista-i-d. (Anexo 1).

El sistema de evaluación de los artículos es "doble ciego", por lo cual los autores no conocerán a los evaluadores ni viceversa, garantizando la objetividad del proceso. Una vez revisados los criterios de fondo y forma de presentación según las normas de la revista $I+D$ Revista de Investigaciones, expuestas a continuación, se enviará el manuscrito a dos pares académicos expertos en el área, quienes elaborarán un concepto científico y de forma del manuscrito en el término de tres meses. Con base en dichos conceptos el editor de $I+D$ Revista de Investigacionesemitirá una decisión editorial en la que se le comunica formalmente al autor los resultados de publicación del artículo que son: aceptado, rechazado, o aceptado con condiciones.

Cuando un artículo es aceptado, los derechos de publicación y reproducción en medios impresos y digitales que permitan el acceso público son de $I+D$ Revista de Investigaciones, bajo licencia de Creative Commons. Sin embargo, se evaluará cualquier petición por parte del autor para obtener el permiso de su reproducción.

\section{Características generales de presentación de los manuscritos.}

Los artículos no deben tener más de 6500 palabras incluidas las referencias, en tipo de letra Times New Roman de 8 puntos con interlineado de 1,0 y márgenes laterales e inferior de $3 \mathrm{~cm}$ x 3,5 superior. Se seguirán en su totalidad las normas para autores estipuladas en $I+D$ Revista de Investigaciones, utilizando procesador de texto Word versión 2007 en adelante. Las tablas o figuras se deben incluir en blanco y negro en el archivo Word y, además, adjuntar aparte el archivo original (p. e., .xls, .jpg, .giff).Las fotografías y figuras que no sean originales del autor, deben adjuntar las debidas autorizaciones para su publicación.
Se recomienda la utilización del castellano estándar para publicaciones científicas, evitando el uso de modismos, regionalismos o similares.

Los autores deben guiarse para la elaboración de su manuscrito por las siguientes normas de $I+D$ Revista de Investigaciones:

Título del artículo: Debe sintetizar la idea principal del estudio en los siguientes aspectos: (a) tener un enunciado conciso, explicativo por si solo de lo investigado, (b) mencionar las variables investigadas y la relación entre ellas, (c) indicar en qué sujetos se estudiaron, (d) usar solo palabras imprescindibles: la extensión máxima del título son12 palabras,(e) evitar abreviaturas,(f) colocar letra inicial del título en mayúscula (en español) y para cada palabra principal (si el título es en inglés),(g) ubicarlo en la parte superior de la hoja centrado.

Nombre del autor y afiliación institucional:Seguir la estructura: Nombre Apellido Apellido (b) ubicar los nombres de autores en el orden de contribución en el estudio, (c) no incluir iniciales, títulos ni grados académicos, (d) incluir hasta dos instituciones que hayan financiado su investigación, (e) si no hay filiación institucional, indicar ciudad y dirección de residencia del autor.

Datos de identificación del estudio: A pie de página en correspondencia con cada autor indique: (a) Título profesional de pregrado, universidad; título más alto de posgrado logrado a la fecha, universidad; nombre del grupo de investigación del investigador, institución, ciudad, dirección electrónica institucional del autor, (b) el enfoque de la investigación,(c) si es un proyecto en curso o culminado,(d) área y subárea del conocimiento al cual pertenece el estudio (ver documento adjunto), (e) nombre del grupo de investigación y su clasificación en COLCIENCIAS, (f) nombre de la institución financiadora de su estudio,(g)Dirección postal y electrónica de la institución financiadora y su PBX,(h) fecha de inicio y terminación del proyecto.

Forma de citación del artículo: Indique: (a) en orden de contribución, por autor la identificación de su artículo así: Primer apellido, letra inicial en mayúscula del primer nombre del primer autor. Haga lo mismo con los nombres de los demás autores agregando antes del último de ellos el símbolo \&y luego el año entre paréntesis; (b) coloque el título de su trabajo como en el título,(c) indique en cursiva el nombre de la revista, seguido por una coma y luego, (c)el número del volumen y entre paréntesis el número de la publicación seguido por coma y separados por guión, los números de página.

Resumen: Condensar en máximo 150 palabras los 
aspectos más relevantes respecto a la metodología, los resultados y las conclusiones del estudio realizado. Escríbirlo en una página nueva con la leyenda "Resumen" en la parte superior izquierda. Escriba el resumen en un solo bloque sin sangría. Al finalizar escriba máximo 5 palabras clave que se encuentren en el tesauro UNESCO. Si elige otro tesauro de su área específica del conocimiento indique su nombre entre paréntesis después de la quinta palabra clave. Haga la versión en inglés del mismo resumen y palabras clave usando un servicio de traducción técnica. Dependiendo del tipo de estudio que haya realizado revise a continuación las normas para construir el resumen, esto mismo le indicará la estructura que debe tener el artículo según el tipo de estudio realizado:

(a)resumen de estudio empírico: Debe contener lo esencial de los siguientes componentes en frases cortas en máximo 150 palabras: (a) el problema que se investiga, (b) el objetivo, (c)los participantes, su edad, sexo, clúster (en el caso de animales, los géneros y especies),(d)metodología, (e)resultados más destacados (indique en valores estadísticos: tamaños del efecto, intervalos de confianza y niveles de significancia estadística, (f) conclusiones, implicaciones y aplicaciones.

(b)resumen de meta-análisis o reseña: Debe contener lo esencial de los siguientes componentes en frases cortas en máximo 150 palabras: (a) el problema 0 relación (es) que se investigaron, (b) los criterios de elegibilidad del estudio, (c) tipos de participantes de los estudios principales,(d) resultados principales (tamaños de efecto relevantes y los moderadores sobresalientes de esos tamaños de efecto), (e) conclusiones que incluyan las limitaciones, vacíos, fallas); y las implicaciones para la teoría, la metodología, la práctica, las políticas, entre otras.

(c)resumen de estudio de orientación teórica: Debe contener lo esencial de los siguientes componentes en frases cortas en máximo 150 palabras: (a) funcionamiento de la teoría, modelo o principios del problema en cuestión, (b) vínculos con los resultados empíricos, (c) relación con la realidad contextual universal y nacional,(d) vínculos con nuevas propuestas 0 aportes novedosos, (f) conclusiones, implicaciones.

(d)resumen de estudio metodológico: Debe contener lo esencial de los siguientes componentes en frases cortas en máximo 150 palabras: (a) tipo general de método que se propone, (b) características esenciales del método planteado, (c) rango de aplicación del método propuesto $y$, en el caso de procedimientos estadísticos, algunas características clave, tales como,(d) robustez y poder de eficiencia.

(e)resumen de estudio de caso: Debe contener lo esencial de los siguientes componentes en frases cortas en máximo 150 palabras: (a) tema y las características relevantes del individuo u organización que se presenta, (b) naturaleza del problema o solución ilustrados mediante el ejemplo de caso, y, (c) las preguntas en relación con alguna otra investigación o teoría adicional.

Títulos y encabezados en el documento: (a) primer nivel: centrado, en negrita, mayúsculas y minúsculas, (b) segundo nivel: alineado a la izquierda en negritas con mayúsculas y minúsculas, (c) tercer nivel: encabezado con sangría, negritas, minúsculas y punto final, (d) cuarto nivel: encabezado con sangría, negritas, cursivas, minúsculas y punto final, (e) quinto nivel: encabezado de párrafo con sangría, cursivas, minúsculas y punto final.

Introducción: En máximo 700 palabras redacte el texto, de lo que se hizo en su estudio y por qué, de tal forma que contenga los siguientes elementos: (a) por qué es importante el problema,(b)cómo se relaciona el estudio con estudios anteriores en el área, en qué difiere, qué aporta con respecto al estado de la cuestión,(c) cuáles fueron las hipótesis y el objetivo primarios del estudio y cómo se relacionan con la teoría y las variables de estudio,(d) cómo se relacionan entre si las hipótesis con el diseño de investigación,(e) cuáles son las implicaciones teóricas y prácticas del estudio, (f)utilice frases cortas, concisas, con alto contenido en cada palabra, que sean específicas, de tipo técnico y en idioma español estándar.

Método: En esta sección se detalla en 1400 palabras como máximo, cómo se realizó el estudio rotulando cada componente por separado, y debe contener: (a) el tipo de estudio, (b) participantes: descripción de las características (edad, género, estrato, nivel educativo, entre otras variables), (c) el proceso de selección y asignación a grupos si fuere pertinente, criterios de inclusión, exclusión, tamaño y precisión. Indique porcentajes, medias; contextos de localización y contacto de los participantes en el estudio, utilización de consentimientos informados. Describa los aspectos legales y éticos tenidos en cuenta para su estudio (leyes, resoluciones, actas de comité de ética de su institución que avalan su estudio). Cambie el término a sujetos cuando la investigación sea con animales e indique datos de género, especie, variedad, familia, número de cría, proveedor, procedencia, tamaños, pesos, estado fisiológico y características y demás que sean pertinentes), (d) instrumentos: Describa los nombres de cuestionarios, pruebas, tests estandarizados utilizados en su estudio, sus propiedades psicométricas. Si es un instrumento traducido aporte datos de validez de traducción. Cuando se trata de estudios aplicados o tecnológicos describa los materiales y aparatos con sus marcas, modelos, series, referencias, valores de calibración y funcionamiento, entre otros que se hayan requerido para el estudio, ubicando el símbolo de marca correspondiente según el caso (XXX®); YYYC),(e) procedimiento: indicar el diseño de investigación utilizado, hacer la descripción detallada, secuencial o jerárquica de las acciones o pasos seguidos para realizar el estudio, el experimento o la intervención y las manipulaciones de sujetos, participantes, materiales y aparatos que ello 
implicó. Dé suficientes detalles de tal forma que el lector interesado pueda replicar las manipulaciones realizadas. $\mathrm{Si}$ se trata de un experimento puede dividir el procedimiento en subsecciones al igual de si se trata de varios experimentos. Indique la manera como se recolectaron los datos,(f) si se trata de una intervención educativa o similar, describa quien la administró, sus características, el número de administradores, (g) señale el contexto donde se realizó el procedimiento, experimento o intervención, cantidad y duración de las mismas, número de sesiones, series, ensayos, eventos, encuentros en que se realizó el procedimiento.

Resultados: Se refieren específicamente a los hallazgos obtenidos en el estudio resultantes del procesamiento de los datos en el caso de los estudios empíricos y deben ser específicos, explícitos y claros. Deben usarse recursos para su visualización tales como: Tablas, figuras, fotografías, entre otros. Estas deben estar identificadas en la parte superior con la leyenda de los que describen y deben ser tan claras que puedan explicarse a si mismas con solo muy poco texto complementario del autor. En la parte inferior deben tener una breve descripción, la fuente de donde se extrajeron y la autorización para su reproducción. En 2000 palabras como máximo incluya la información más relevante, pertinente y suficiente de su estudio contemplando lo siguiente: (a) tamaños de efecto obtenidos grandes o pequeños sin omitir datos en contra de lo esperado o incómodos, (b) fechas de reclutamiento y períodos de seguimiento de los participantes o sujetos, (c) intervalos de confianza,(d) los tamaños de las muestras, sus medias, desviaciones estándar, las varianzas, (e) en los casos en que se requiera, los datos esenciales de: los análisis multivariados, de regresión, de modelos de ecuaciones estruturales y el modelo lineal jerárquico, las matrices de correlación, (f) para los estudios en muestras pequeñas o de caso único proporcione los datos brutos en tablas completos para ulteriores meta-análisis, $(\mathrm{g})$ para las pruebas estadísticas inferenciales (como pruebast, F.o pruebas $\mathrm{X}^{2}$ ), incluya lamagnitud obtenida o el valor del estadístico de prueba, los grados de libertad, la probabilidad (Valor $p$ exacto), el tamaño y la dirección del efecto, (h) cuando presente estimaciones puntuales (como medias dela muestra o coeficientes de regresión), siempre incluya una medición asociada de variabilidad(precisión), con una indicación del error estándar,(i) incluya intervalos de confianza especialmente de $95 \%$ y $99 \%$,(j) mencione resultados adversos con consecuencias o efectos secundarios del estudio realizado.

Discusión y Conclusiones: Alude a la discusión estricta sobre los resultados obtenidos entorno a las preguntas de investigación y los estudios previos, desde lo cual se formulan las conclusiones del estudio. En 1400 palabras como máximo debe contener: (a) las fuentes de sesgo potencial y otras amenazas a la validez interna que presenta su estudio, (b) la imprecisión de las mediciones que se pudo presentar, (c) la cantidad general de pruebas o la superposición entre pruebas que pudo haber, (d) los tamaños del efecto observados vs los esperados y las explicaciones alternativas, así como los alcances de generalización o validez externa de sus resultados, (e) el cumplimiento de las hipótesis ya sea parcial, deficitario o nulo y los argumentos al respecto en función de estudios anteriores,(f) limitaciones o debilidades del estudio con respecto a los estudios antecedentes; barreras encontradas, (g) indique aspectos, intensidad, magnitud en que fue exitoso su estudio con respecto al objetivo de la investigación, sus consecuencias teóricas, metodológicas y prácticas y lo que queda sin resolverse.

Agradecimientos: En 100 palabras indique fuentes de financiación, personas, instituciones que hayan contribuido a la realización de su trabajo de manera formal. Proporcione información específica sobre actas de aprobación de presupuesto para su estudio, pagos específicos de subvenciones para los investigadores y otro tipo de ayudas significativas sin las cuales el proyecto no hubiese sido una realidad. Menciones agencias internacionales, nacionales o locales públicas o privadas que intervinieron respaldando su proyecto en alguna forma sustancialmente eficaz.

Citas y Referencias: Se refiere a los libros, artículos científicos y demás fuentes de consulta que avalan el estudio realizado. Estas deben ser mínimo 35 y máximo 60 y deben corresponder de manera exacta las contenidas intratexto con la lista de las mismas al final del documento. Debe tenerse en cuenta el formato de con normas APA sexta edición.

Ética de la publicación en $I+D$ Revista de Investigaciones.

La Revista $I+D$ Revista de Investigaciones se acoge a los lineamientos del Código Deontológico y Bioético del Ejercicio de la investigación. Para la publicación de manuscritos en esta revista es necesario que el(los) autor(es) garantice(n) el cumplimiento de los principios éticos mencionados en la "Declaración de cumplimiento de los principios éticos",el cual podrá ser descargado del link http://www.udi.edu.co/investigaciones/102-revista-i-d. (Anexo 2).

Declaración sobre Conflicto de Intereses.

Dando cumplimiento a los requerimientos para la publicación de artículos en la Revista $I+D$ Revista de Investigaciones es necesario que los autores informen, preferiblemente como nota de autor en el manuscrito, los posibles conflictos de interés en el trabajo de investigación para lo cual deben diligenciar el documento diseñado para este fin, el cual podrá ser descargado del link http://www.udi.edu.co/investigaciones/102-revista-i-d. (Anexo 3). 


Bucaramanga Calle 9 No. 23 - 55 PBX: 6352525

\section{Barrancabermeja} Calle 48 No. 14 - 61 PBX: 6222863

San Gil Carrera 9 No. 10 - 40 Tel. 7242224

\section{Ediciones}

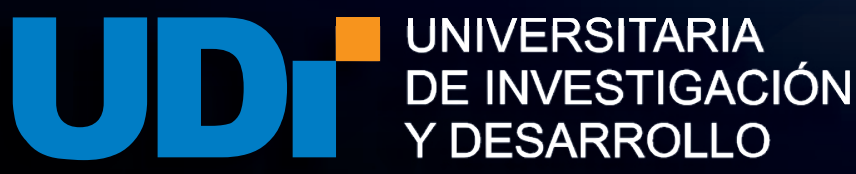

www.udi.edu.co 